\title{
Contraintes à l'adoption des bonnes pratiques de Gestion Durable des Terres dans les zones soudaniennes et soudano-sahéliennes du Burkina Faso
}

\author{
Edmond N. KOHIO ${ }^{1 *}$, Alassane G. TOURE ${ }^{2}$, Michel P. SEDOGO ${ }^{3}$ et \\ Karimou J-M. AMBOUTA ${ }^{4}$ \\ ${ }^{1}$ Centre Régional AGRHYMET, BP 11011, Niamey, Niger. \\ ${ }^{2}$ Division du Partenariat et de la Coordination des Conventions Internationales en matière d'Environnement \\ (DPCIE), Secrétariat Permanent du Conseil National du Développement Durable (SP-CNDD), 01 BP 6486 \\ Ouagadougou 01, Burkina Faso. \\ ${ }^{3}$ Laboratoire Sol-Eau-Plante, Département Gestion des Ressources Naturelles/Systèmes de Production \\ (GRN/SP), Institut de l'Environnement et de Recherche Agricole (INERA), 01 BP 476 Ouagadougou 01, \\ Burkina Faso. \\ ${ }^{4}$ Département Science du sol, Faculté d'agronomie, Université Abdou Moumouni de Niamey, BP 10960, \\ Niamey, Niger. \\ *Auteur correspondant, E-mail : kedmondd@gmail.com; Tél. : (+226) 70970744.
}

\section{REMERCIEMENTS}

Les auteurs remercient le Centre Régional AGRHYMET qui a financé cette étude.

\section{RESUME}

La dégradation des terres est une problématique majeure à laquelle est confrontée l'ensemble des pays du Sahel. Relever le défi lié à la gestion durable des ressources naturelles passe par l'utilisation des bonnes pratiques de gestion durable des terres (GDT). Cependant, l'adoption de ces pratiques est confrontée à diverses contraintes. La présente étude vise à mieux cerner ces contraintes en vue de favoriser l'adoption des bonnes pratiques de GDT. Pour ce faire, une enquête a été menée auprès de soixante (60) paysans pilotes de la région du Centre Ouest du Burkina Faso. Les résultats montrent que les contraintes matérielles et financières sont présentes aussi bien dans la zone soudanienne que dans la zone soudano-sahélienne. Les contraintes techniques, celles liées à la mauvaise organisation des marchés agricoles et à l'insécurité foncière sont nettement plus prononcées dans la zone soudanienne, contrairement à la zone soudano-sahélienne. Une adoption d'envergure des bonnes pratiques de GDT pourrait favoriser une meilleure gestion des ressources naturelles et asseoir les fondements d'un développement économique et social durable. Toutefois, une véritable prise de conscience des acteurs, assortie d'un accompagnement conséquent et coordonné, s'avère nécessaire. (c) 2017 International Formulae Group. All rights reserved.

Mots clés : Dégradation des terres, bonnes pratiques, milieu paysan, zones climatiques, pays du Sahel, Burkina Faso.

\section{Constraints to the adoption of good practices of sustainable land management in sudanian and sudano-sahelian climatic zones of Burkina Faso}

\author{
ABSTRACT \\ Land degradation (LD) is a major issue in countries of the Sahel region in West Africa. Meeting the \\ challenge of sustainable natural resource management requires the use of sustainable land management (SLM)
}


practices. However, adoption of these practices is subjected to various constraints. The purpose of this study was to identify these constraints in order to promote the adoption of good SLM practices. To do this, a study was carried out among sixty (60) pilot farmers from the Western central Region of Burkina Faso. Results show that material and financial constraints are both present in sudanian and in sudano-sahelian climatic zones. Technical constraints as well as those related to poor organization of agricultural markets and those due to land insecurity are more pronounced in sudanian area than in sudano-sahelian area. Widespread adoption of good SLM practices could lead to better management of natural resources and provide the basis for sustainable economic and social development. However, a real awareness of the actors along with a consistent and coordinated follow-up are necessary.

(C) 2017 International Formulae Group. All rights reserved.

Keywords: Land degradation, good practices, farmer environment, climatic zones, Sahel region country, Burkina Faso.

\section{INTRODUCTION}

L'appauvrissement progressif des sols du fait de l'agriculture minière (Bationo et al., 1998) et la pression liée à la recherche des moyens de subsistance obligent les paysans du Burkina Faso à étendre les superficies cultivées à des terres marginales (Traoré et Toé, 2008). Les sols, ainsi soumis à une forte pression anthropique, en plus des conditions climatiques de plus en plus défavorables, deviennent très sensibles à la dégradation et ne supportent pas, de façon soutenue, les systèmes et modes de production agricole actuellement pratiqués. La performance du secteur agricole est de ce fait médiocre et fortement influencée par la faible productivité des terres. Ainsi, la progression de la dégradation des terres menacerait, non seulement, l'approvisionnement alimentaire des populations humaines, mais contribuerait également aux changements climatiques (SP-CONEDD, 2006).

Dans un tel contexte, relever les défis liés à la sécurité alimentaire et au changement climatique implique nécessairement une transformation profonde des modes de production. Les bonnes pratiques de Gestion Durable des Terres (GDT), qui permettent d'accroître la teneur en carbone du sol, offrent de nouvelles alternatives (FAO, 2011). Ces pratiques favorisent une gestion intégrée de la fertilité des sols qui permet d'obtenir une amélioration du taux de matière organique et de la structure des sols, contribuant ainsi à l'augmentation de l'infiltration et à la réduction de l'érosion (CILSS, 2012 ; GIZ, 2012). Ces effets bénéfiques peuvent induire une augmentation des rendements des cultures (Scopel et al., 2005 ; Savadogo et al., 2011), pouvant atteindre 170\% (WOCAT, 2009).

Malgré les efforts fournis par les services techniques dans la vulgarisation de ces bonnes pratiques qui, d'ailleurs, semblent à la fois pertinentes et performantes, leur adoption demeure toujours faible dans le Centre Ouest du Burkina Faso. L'objectif de la présente étude est d'identifier les éventuelles contraintes à l'origine de cette faible adoption.

\section{MATERIEL ET METHODES}

\section{Zone d'étude}

L'étude a été conduite dans six communes (Léo, To, Dalo, Poa, Kindi et Didyr) de la région du Centre Ouest du Burkina Faso (Figure 1). La région s'étend sur la zone climatique soudanosahélienne entre les isohyètes 900 et $600 \mathrm{~mm}$, dans la partie nord et la zone climatique soudanienne bordée par l'isohyète $900 \mathrm{~mm}$ et l'isohyète $1100 \mathrm{~mm}$, au sud de la région (PNUD, 2013). Le climat 
est caractérisé par une saison sèche et une saison pluvieuse, avec une pluviométrie capricieuse et irrégulière. Les sols sont principalement des Gleysols et des Lixisols, pauvres en matière organique et très sensibles à l'érosion, quand ils ne sont pas protégés. L'utilisation continue des sols a entrainé la dégradation extrême de ses potentialités physiques et chimiques. La végétation est essentiellement caractérisée par trois types de formations végétales du nord vers le Sud, à savoir : une savane arbustive, une savane arborée et des forêts galeries ou claires. La région est densément peuplée ; on y compte en moyenne 31 habitants $/ \mathrm{km}^{2}$ dans la zone soudanienne et 92 habitants $/ \mathrm{km}^{2}$ dans la zone soudano-sahélienne, dont l'activité dominante est la production agro-sylvo-pastorale.

La région du Centre Ouest a été choisie comme zone d'étude du fait de sa situation agroclimatique présentant à la fois une zone soudanienne et une zone soudano-sahélienne. Cette répartition climatique a été considérée comme une typologie à partir de laquelle les comparaisons ont été faites. Les raisons de cette typologie étaient que le niveau de dégradation des terres, les pratiques de gestion durable des terres qui en découlent et les contraintes liées à l'adoption de ces bonnes pratiques peuvent varier en fonction du type de climat. Ainsi, à partir de la méthode d'échantillonnage en grappe, six (6) communes, à raison de trois (3) communes par zone climatique, ont été retenues, en considérant tour à tour, (i) la présence et la disponibilité des paysans pilotes, (ii) la disparité géographique et socio-économique des communes dans chaque zone climatique, ainsi que (iii) l'accessibilité des communes.

\section{Identification des contraintes à l'adoption des bonnes pratiques de GDT}

L'échantillonnage des paysans pilotes a été fait à partir des bases de données des structures techniques intervenant dans la GDT. Au niveau de la zone soudanienne, il s'est agi de faire un recensement des paysans pilotes disponibles (au total 30 paysans pilotes dans les trois communes), compte tenu de leur nombre assez limité. Pour le cas de la zone soudano-sahélienne, un échantillon de paysans pilotes a été retenu aléatoirement à partir de la base de données et proportionnellement au nombre des paysans pilotes retenus dans la zone soudanienne. Pour ce faire, la méthode de sondage aléatoire simple a été retenue (tirage sans remise selon la norme statistique à $95 \%$ d'intervalle de confiance).

A l'aide de l'échantillon de paysans pilotes, une enquête socio-économique a été conduite. Elle a d'abord consisté en une phase d'essai du questionnaire, qui a permis d'adapter ledit questionnaire aux réalités du terrain, et de prendre en compte les suggestions des paysans. L'enquête proprement dite a été ensuite effectuée auprès des paysans pilotes retenus. Cette enquête s'est appuyée sur deux (2) outils.

Le premier est un questionnaire ou interview structuré. Il contenait l'identification des paysans pilotes, les données sur les activités socio-économiques et les éventuelles contraintes à l'adoption des bonnes pratiques de GDT. Le second, s'est appliqué à des Interviews Non Structurées (INS), sans questionnaire ni guide d'entretien, mais ayant trait au sujet de l'étude, qui sont intervenues de façon fortuite, à des occasions propices avec des acteurs directs ou indirects. Elles nous ont permis de prendre en compte certaines observations sur l'organisation des acteurs, les approches et les non-dits. 


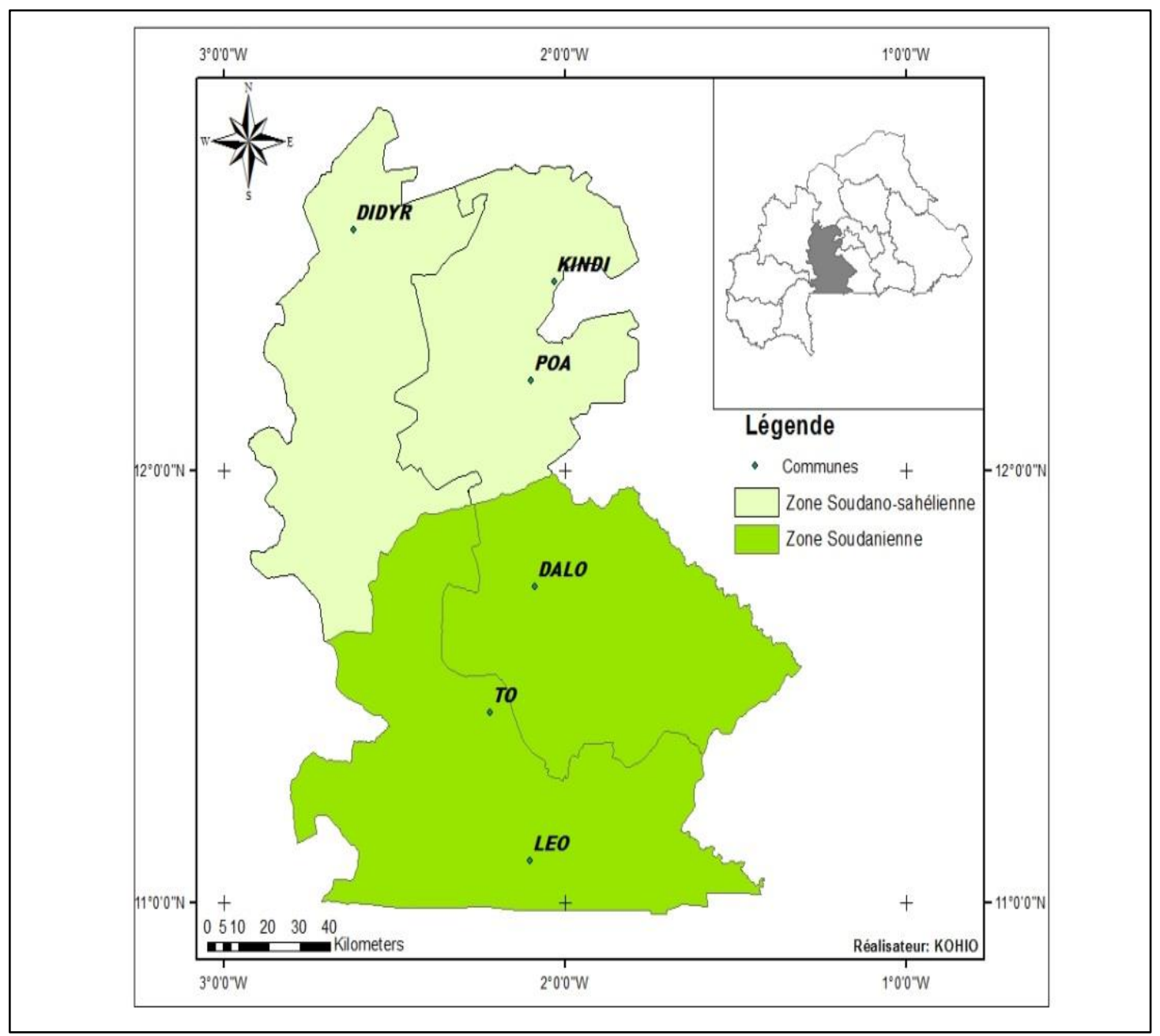

Figure 1 : Carte de la zone d'étude.

\section{RESULTATS}

Les résultats montrent que les contraintes matérielles et financières sont fortement présentes aussi bien dans la zone soudanienne que dans la zone soudanosahélienne pour, respectivement, 95 et $78,33 \%$ des paysans enquêtés (Figure 2). Les contraintes techniques sont plus importantes dans la zone soudanienne (60\%) que dans la zone soudano-sahélienne (10\%). Les contraintes liées à la mauvaise organisation des marchés et à l'insécurité foncière ont moins d'influence sur la non adoption des bonnes pratiques, quoique plus prononcées en zone soudanienne qu'en zone soudanosahélienne, avec des taux évalués à 43,33 et
$36,67 \%$ pour la zone soudanienne contre 3,33 et $6,67 \%$ pour la zone soudano-sahélienne. Les résultats montrent aussi que les pesanteurs sociales ne sont pas des contraintes majeures à l'adoption des bonnes pratiques de GDT. Dans la zone soudanienne, d'autres contraintes sont ressorties des Interviews Non structurées (INS), telles la non prise de conscience de certains paysans sur la dégradation des terres, la disponibilité de terres cultivables chez certains paysans et le manque d'assurance quant à la capacité des bonnes pratiques de GDT à offrir des solutions palliatives. 


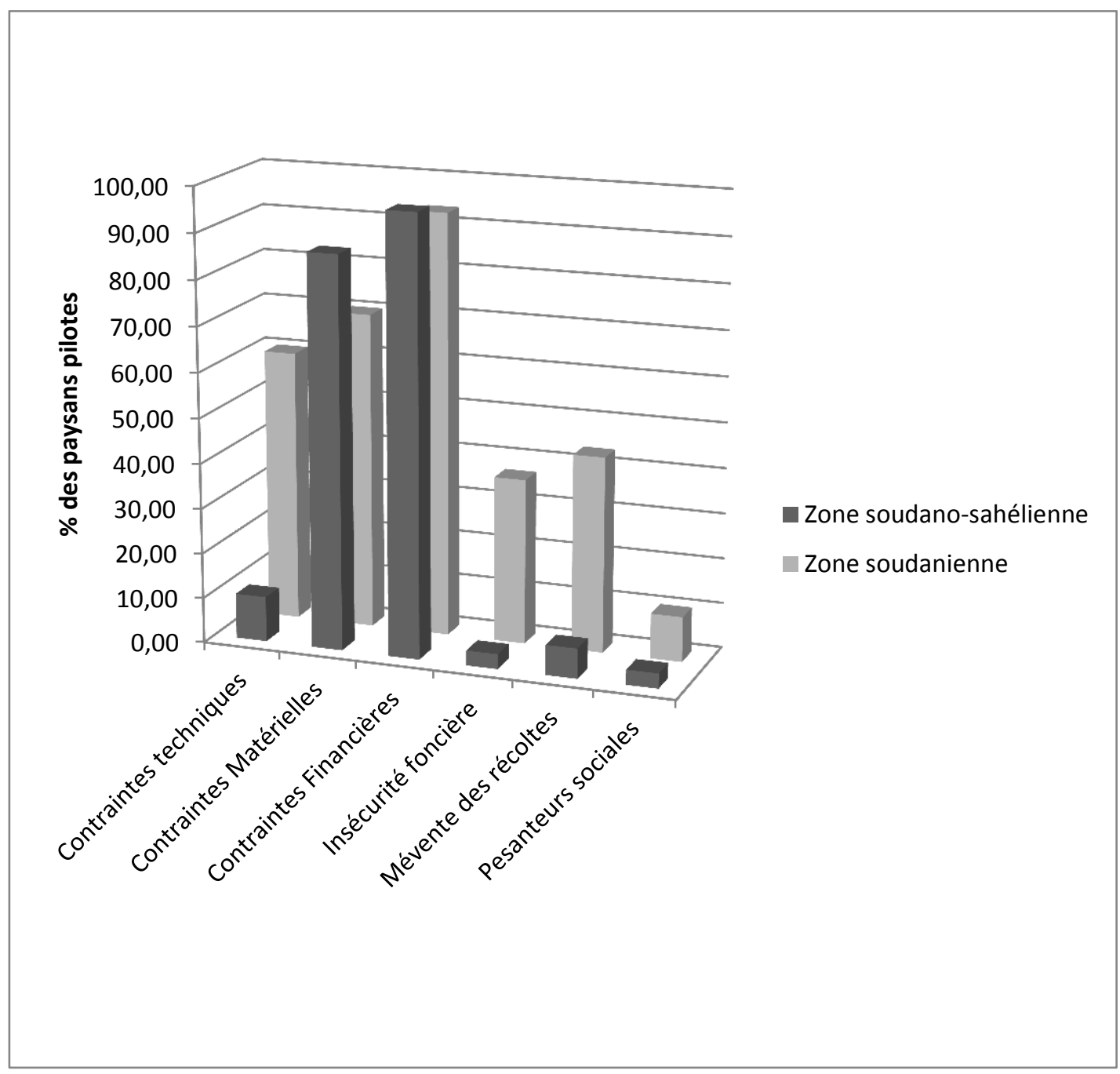

Figure 2 : Contraintes à l'adoption des bonnes pratiques de GDT selon les paysans.

\section{DISCUSSION}

Comme on pouvait s'y attendre, l'adoption des bonnes pratiques de gestion durable des terres est freinée par diverses contraintes, dont les plus importantes, d'ordres matériel et financier, pourraient s'expliquer par le niveau de pauvreté très élevé de ces populations rurales (PNUD, 2013). Ces résultats sont en parfaite concordance avec ceux obtenus par Deffo et al. (2004) sur l'adoption du système maïsmucuna-engrais minéraux et par Kini (2007) en zone soudano-sahélienne du Burkina Faso. Selon ce dernier, le coût de réalisation des techniques de conservation de l'eau du sol est le facteur le plus déterminant à leur adoption par les ménages agricoles. Ces résultats corroborent également ceux de Zoungrana (2013) en ce qui concerne la contrainte matérielle, mais en diffèrent pour le cas spécifique de la contrainte financière. Cette différence notoire pourrait découler, à la fois, du niveau variable de pauvreté des ménages agricoles et de la différence relative des coûts de main-d'œuvre en fonction des localités. Les contraintes techniques sont plus présentes en zone soudanienne, contrairement à la zone soudano-sahélienne. La dégradation des 
terres, plus prononcée en zone soudanosahélienne, aurait donc contraint ces populations à apprendre les pratiques résilientes.

Par ailleurs, nos résultats montrent que l'insécurité foncière n'est pas une grande menace à l'adoption des bonnes pratiques de GDT en zone soudano-sahélienne. Ce qui contraste avec ceux de FAO (2007) qui avait trouvé que l'insécurité foncière constituait une contrainte majeure à l'adoption des bonnes pratiques agricoles en zone soudanienne du Burkina Faso. Une tentative d'explication du contraste nous est donnée par un groupe de paysans rencontrés à Poa, selon lesquels, l'adoption des bonnes pratiques de GDT en zone soudano-sahélienne répond plus à un besoin impératif de production qu'une volonté manifeste de gérer durablement les terres. De ce fait, même si le paysan n'est pas sûr de pouvoir produire sur la même terre les années suivantes, il est amené à adopter le minimum de bonnes pratiques pour assurer la production actuelle. En outre, la valeur assez importante de la contrainte liée à l'insécurité foncière en zone soudanienne, caractérisée par des conditions pédo-climatiques relativement plus favorables, pourrait contribuer à consolider cette thèse. Ce serait aussi cette difficulté de production en zone soudano-sahélienne en l'absence de toutes mesures de GDT, qui expliquerait que la contrainte liée à la mauvaise organisation des marchés agricoles et des chaînes de valeur soit moins prononcée dans cette zone, comparativement à la zone soudanienne au niveau de laquelle cette contrainte est plus marquée. La priorité pour les paysans de la zone soudano-sahélienne étant l'assurance de la sécurité alimentaire et non la recherche de profits. Traoré et Toé (2008) ont trouvé des résultats différents selon lesquels, les insuffisances au niveau de la commercialisation des produits agricoles ainsi que la faible structuration des filières constituaient des freins importants à l'adoption des bonnes pratiques agricoles.
Ainsi, le but de la production est aussi un facteur important qui peut influencer l'adoption des bonnes pratiques.

Les Interviews Non structurées (INS) avec les paysans enquêtés ont révélé l'existence d'un rapport hiérarchique entre les différentes contraintes. Ce rapport présente une configuration différente suivant les zones. Ainsi, en zone soudano-sahélienne, les paysans sont d'abord confrontés à la contrainte technique, même si cette contrainte ne concerne que seulement $10 \%$ des cas. Ensuite, viennent les contraintes matérielles et financières. En zone soudanienne, la chaîne des contraintes est relativement plus longue. D'abord, une frange des paysans n'est pas consciente de la dégradation de leurs terres. Pour ceux qui en sont conscients, la possibilité de mise en jachère, en lien avec la forte disponibilité de terres cultivables chez certains paysans, constitue un frein à l'adoption des bonnes pratiques de GDT. Même dans le camp des paysans n'ayant pas de possibilités de mise en jachère, certains ne sont toujours pas convaincus de la capacité des bonnes pratiques de GDT à pallier la dégradation des terres. Aussi, pour les paysans qui semblent convaincus des effets bénéfiques des bonnes pratiques, la capacité technique de réalisation de ces pratiques fait-elle souvent défaut. Enfin, pour les plus téméraires qui ont réussi à acquérir la capacité technique, l'absence de moyens matériels et financiers est malheureusement un sérieux obstacle à surmonter.

\section{Conclusion}

On peut globalement retenir que le classement des contraintes montre que le manque d'argent et l'absence de matériels adaptés constituent les contraintes majeures qui limitent l'adoption des bonnes pratiques de GDT. Les exploitations agricoles familiales de la zone soudano-sahélienne sont plus favorables à l'adoption des bonnes pratiques, du fait de la difficulté de pouvoir produire en 
l'absence de toutes mesures de gestion durable des terres. A l'opposé, les terres de la zone soudanienne présentent, par endroits, des capacités de production toujours appréciables. Cette situation ne favorise pas l'adoption des bonnes pratiques de GDT par certains paysans, et vient ainsi renforcer le lot des contraintes, déjà très diversifiées.

Les principaux déterminants de l'adoption des bonnes pratiques de GDT sont donc la disponibilité de ressources matérielle et financière. Dans l'impossibilité de satisfaire ces conditions, il apparaît judicieux de proposer aux paysans des pratiques résilientes qui tiennent compte de ces réalités. Les pratiques les plus abordables du point de vue des exigences matérielles et financières pourraient être les meilleures pistes de solutions à explorer. Pour le cas spécifique de la zone soudanienne, où certains paysans demeurent sceptiques quant à la capacité des bonnes pratiques de GDT à apporter des solutions palliatives à la problématique de dégradation des terres, les champs écoles en milieu paysans pourraient être des solutions envisageables.

\section{CONFLIT D'INTERETS}

Les auteurs déclarent qu'ils n'ont pas de conflits d'intérêts.

\section{CONTRIBUTIONS DES AUTEURS}

ENK a collecté de données, analysé de données, rédigé l'article, AGT a choisi les sites de collecte de données, a créé les facilités administratives de la collecte de données; MPS a choisi les sites de collecte de données décrit les méthodes de collecte de données et a corrigé l'article ; KJ-MA a orienté du travail, et a corrigé l'article.

\section{REMERCIEMENTS}

Les auteurs remercient tous les acteurs impliqués dans cette étude. Nos remerciements s'adressent particulièrement au Sous-programme pour la Région du Centre-
Ouest du Programme National de Partenariat pour la Gestion Durable des Terres au Burkina Faso (CPP-RCOS) pour son assistance technique pendant la collecte de données.

\section{REFERENCES}

Bationo A, Koala S, Ayuk E. 1998. Fertilité des sols pour la production céréalière en zone soudano-sahélienne et valorisation des phosphates naturels. Cahiers Agricultures, 7(5): 365-371. DOI : http://revues.cirad.fr/index.php/cahiersagricultures/article/download/30109/298 69

CILSS. 2012. Bonnes pratiques agro-sylvopastorales d'amélioration durable de la fertilité des sols au Burkina Faso. Document technique. Ouagadougou, Burkina Faso, p. 194.

Deffo1 V, Hounzangbé-Adoté SM, Maliki R, Ferroukh HHMO, Torquebiau E. 2004. Contraintes contingentes à l'adoption d'une technologie. Evaluation contingente des contraintes à la fertilisation des sols par le système maïsmucuna-engrais minéraux au sud du Togo. Tropicultura, 22(1): 19-25. DOI : www.tropicultura.org/text/v22n1/19.pdf

FAO. 2007. Systèmes agraires durables, vulnérabilité et bonnes pratiques agricoles dans l'Ouest du Burkina Faso. Actes de l'atelier FAO-INERA sur les Bonnes Pratiques Agricoles, Bobo Dioulasso, Burkina Faso, 18-20 Mars 2004. Document de travail FAO - BPA 6, Rome, Italie, p. 143.

FAO. 2011. La pratique de la gestion durable des terres. Directives et bonnes pratiques pour l'Afrique subsaharienne : Application sur le terrain. TerrAfrica, WOCAT, FAO. Rome, Italie, p. 249.

GIZ. 2012. Bonnes pratiques de conservation des eaux et des sols : Contribution à l'adaptation au changement climatique et à la résilience des producteurs au Sahel. Bonn, Allemagne, p. 60. 
Kini J. 2007. Analyse des déterminants de l'adoption des techniques de conservation des eaux et des sols dans le plateau central du Burkina Faso. Mémoire DEA, Université de Ouagadougou, Ouagadougou, Burkina Faso, p. 64.

PNUD. 2013. Document de Projet (PRODOC) du Programme de Partenariat Pays pour la gestion durable des terres CPP-GDT : Sous-programme pour la Région CentreOuest. Ouagadougou, Burkina Faso, p. 49.

Savadogo M, Somda J, Seynou O, Zabré S, Nianogo AJ. 2011. Catalogue des Bonnes Pratiques d'Adaptation aux Risques Climatiques au Burkina Faso. UICN: Ouagadougou, Burkina Faso; 52.

Scopel E, Douzet JM, Da Silva FAM, Cardoso A, Aloisio J, Moreira A, Findeling A, Bernoux M. 2005. Impacts des systèmes de culture en semis direct avec couverture végétale (SCV) sur la dynamique de l'eau, de l'azote minéral et du carbone du sol dans les cerrados brésiliens. Cahiers Agricultures, 14(1) : 71-75.

DOI : http://revues.cirad.fr/index.php/cahiersagricultures/article/download/30491/302 51

SP-CONEDD. 2006. Revue scientifique sur l'état de la dégradation des sols du Burkina Faso. Rapport final, Ouagadougou, p. 105.

Traoré K, Toé AM. 2008. Capitalisation des initiatives sur les bonnes pratiques agricoles au Burkina Faso. Rapport d'étude, MAHRH, Ouagadougou, Burkina Faso, p. 83.

WOCAT. 2009. Les Bénéfices de la Gestion Durable des Terres. CDE, Université de Berne, Suisse ; 16.

Zoungrana F. 2013. Etude d'impacts technicoéconomiques à moyen terme de la conservation et de la restauration des sols dans la province du Kourwéogo au Burkina Faso : cas des cordons pierreux et de la fumure organique. Mémoire de Master II, Agrhymet, Niamey, Niger, p. 67. 